\title{
Stabilizing Brillouin Fiber Lasers
}

\author{
$\underline{\text { A.A. Fotiadi }}^{1,2,3, *}$, V.V. Spirin ${ }^{4}$, J.L. Bueno-Escobedo ${ }^{4}$, \\ P. Mégret $^{2}$, D.A. Korobko ${ }^{1}$, I.O. Zolotovskii ${ }^{1}$ \\ ${ }^{1}$ Ulyanovsk State University, Ulyanovsk, Russian Federation \\ ${ }^{2}$ University of Mons, Mons, Belgium \\ ${ }^{3}$ Ioffe Physico-Technical Institute of the RAS, St. Petersburg, Russian Federation \\ ${ }^{4}$ Scientific Research and Advanced Studies Center of Ensenada (CICESE), México \\ "E-mail: Andrei.Fotiadi@gmail.com
}

DOI: 10.31868/RFL2020.96-97

Low-noise lasers are a revolutionary tool in precision spectroscopy, displacement measurements, and the development of advanced optical atomic clocks. Further applications include lidars, coherent communications, frequency synthesis, and precision sensors of strain, motion, and temperature. While all applications benefit from lower frequency noise and robust laser design, some of them also require a simple laser configuration generating two locked frequencies. Recently, we have demonstrated a kHzlinewidth laser just combining a standard DFB laser diode and a few passive telecommunication components [1]. The principle of operation employs the mechanism of selfinjection locking that could significantly improve the DFB laser performance. While a typical linewidth of free-running DFB semiconductor lasers ranges from a few to tens $\mathrm{MHz}$, self-injection locking of the DFB laser through an external fiber ring cavity causes a drastic reduction of the laser linewidth down to a few $\mathrm{kHz}$ making it attractive for a new range of applications.

In such a laser configuration, the same external fiber cavity can be used for selfinjection locking of the DFB laser and as Brillouin scattering media to generate the Stokes shifted optical wave. It is worth noting that other teams have already used the fiber ring cavity to generate Brillouin wave from an external laser diode [2]. However, they always face the problem of coupling between the DFB laser and the ring fiber cavity that is, in general, technically complicated and cost-consuming tasks. In our approach, the implementation of the self-injection locking mechanism in the Brillouin fiber laser configuration allows to maintain natural coupling between the DFB laser and the external fiber cavity enabling dual-frequency laser operation. However, a stable generation of two frequencies by the self-injection locked DFB laser has not been achieved yet, making it unavailable for many prosperous applications [3].

Several approaches have been performed to stabilize the laser operation in the self-injection locking regime. The main drawback of this technique is its high sensitivity to fluctuations of the configuration parameters and surroundings. Once getting locked to the cavity resonance, the laser starts to generate the cavity resonant frequency. Then any slow change of the ring mode frequency (due to environment temperature fluctuations, for example) near the stability point causes the same change of the laser frequency. However, a more extended drift of the cavity mode frequency $(>10 \mathrm{MHz})$ causes mode-hopping making laser operation temporally unstable. As a result, a stable laser operation is commonly observed for a few seconds. With precise stabilization of the laser diode current and temperature used in conjugation with the thermal stabilization of the whole fiber configuration the periods of laser stability could be extended to tens of minutes and even more. However, such stabilization method is technically complicated and cost-consuming. The use of polarization maintaining fibers improves to some extent the laser stability preventing polarization mode-hopping [4].

Alternatively, stabilization of semiconductor DFB laser in self-injection locking regime could be achieved by implementing an active optoelectronic feedback con- 
trolled by a low-cost USB-DAQ card [5]. In this approach, the narrowing of DFB laser linewidth is still provided by self-injection locking, whereas the active feedback is used only to maintain the laser operation in this regime. Therefore, in terms of feedback circuit bandwidth, complexity, and allocated memory, this solution is much less consuming than optoelectronic systems commonly used with fiber lasers.

Here, we introduce a simple dual-frequency laser [Fig.1, a] leveraging a ring fiber cavity that exploits one ring cavity mode for self-injection locking of a standard semiconductor DFB laser to generate light at pump frequency and another cavity mode to generate Stokes light via stimulated Brillouin scattering. In contrast to the previous laser configurations [3], the system is supplied with a low-bandwidth active optoelectronic feedback helping to maintain the regime of self-injection locking that in its turn takes charge of stable operation of two mutually locked pump and Stokes frequencies [Fig.1, b]. This configuration reduces the natural Lorentzian linewidth of the light emitted by the laser at pump and Stokes frequencies down to $400 \mathrm{~Hz}$ and $300 \mathrm{~Hz}$, respectively, and features a stable $300-\mathrm{Hz}$-width RF spectrum of beating between two laser outputs [Fig.1, c]. Specifically, the laser cavity is spliced from standard SMF-28 components, has no thermal control of the fiber configuration, and employs a DFB laser diode powered by a standard driver. In future, translating the proposed laser design to integrated photonics will dramatically reduce cost and footprint for many applications such as ultra-high capacity fiber and data center networks, atomic clocks, and microwave photonics.

b)
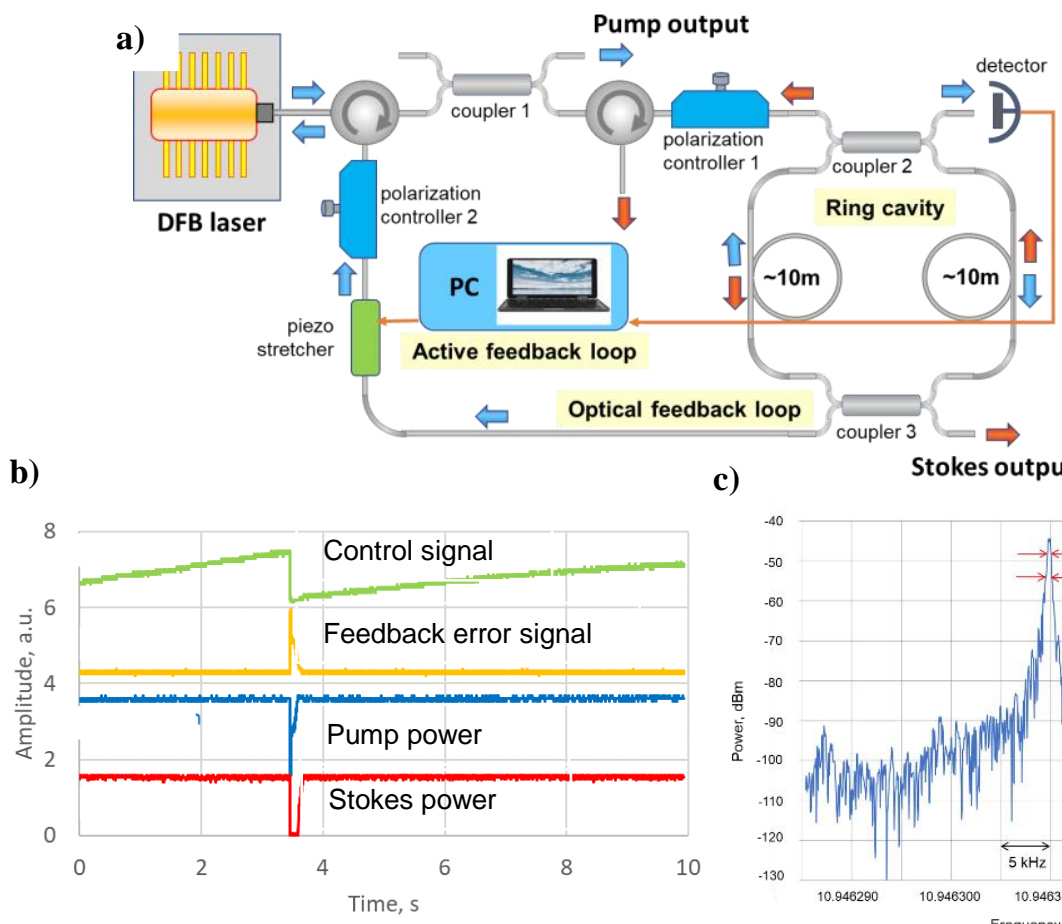

c)

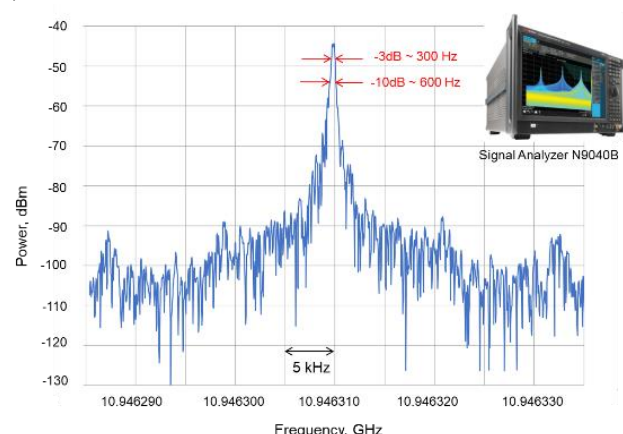

Fig. 1. Dual-frequency laser: experimental setup (a), output powers and operation of optical and electronic feedbacks against a knock on the fiber cavity (b), RF spectrum of beating between two frequencies.

The work is supported by the Russian Science Foundation (grant № 18-12-00457).

\section{References}

[1] C. A. López-Mercado et al., Optics Communications 359, 195-199 (2016).

[2] L. Rossi et al., Opt. Express 27, 29448-29459 (2019).

[3] V. V. Spirin et al., Laser Physics Letters 10, 015102 (2013).

[4] J. L. Bueno Escobedo et al., Results in Physics 12, 1840-1842 (2019).

[5] V. V. Spirin et al., Opt. Express 28, 478-484 (2020). 